\title{
Degenerações periféricas da retina em pacientes candidatos à cirurgia refrativa
}

\author{
Peripheral retina degeneration in patients who arecandidates for refractive surgery
}

Paulo Henrique de Avila Morales ${ }^{(1)}$

Michel Eid Farah ${ }^{(2)}$

Ana Luisa Höfling'-Lima ${ }^{(3)}$

Norma Alleman ${ }^{(3)}$
Pedro Paulo Bonomo ${ }^{(3)}$

\begin{tabular}{|c|}
\hline RESUMO \\
\hline 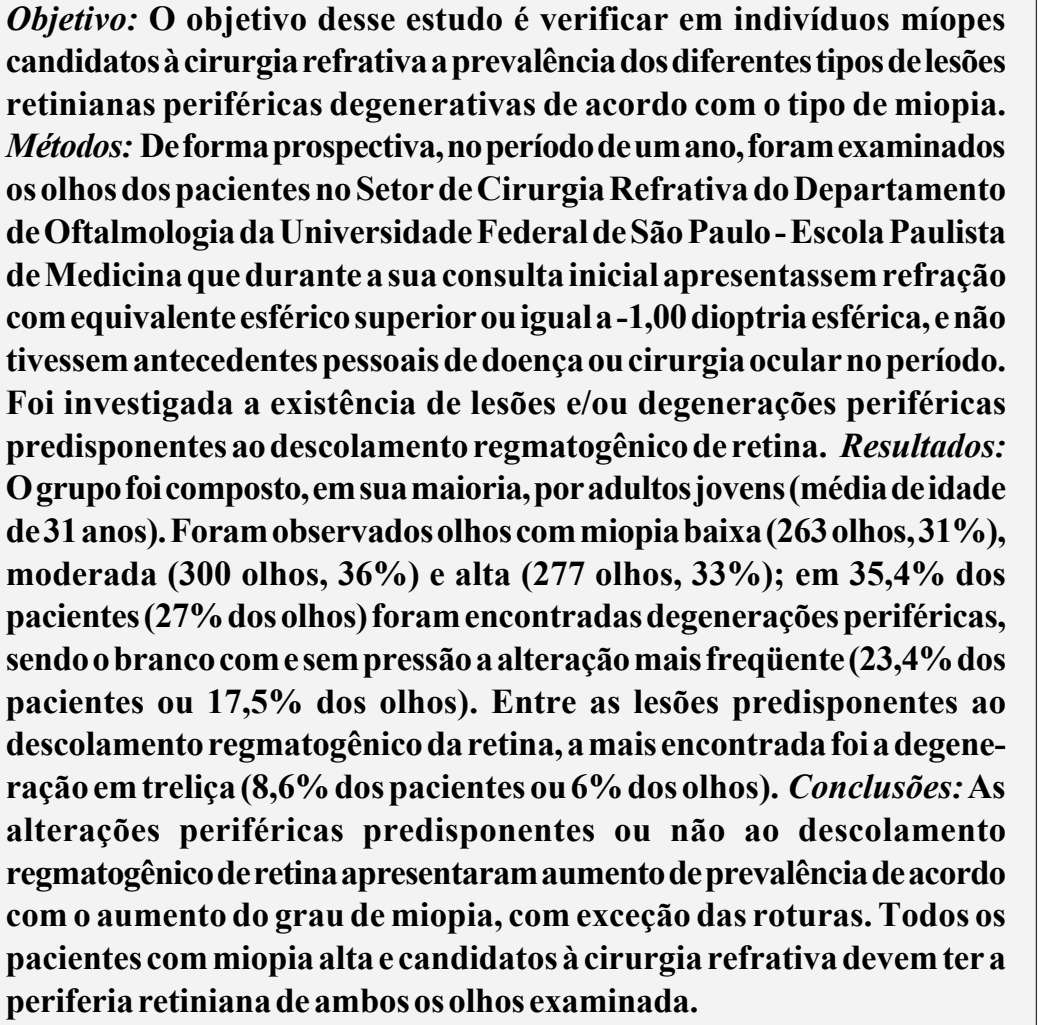 \\
\hline
\end{tabular}

Descritores: Descolamento retiniano; Ceratectomia fotorrefrativa por excimer laser/ efeitos adversos; Miopia/cirurgia.

gia da Universidade Federal de São Paulo - Escola Paulista de Medicina (EPM), com apoio da Fundação de Amparo à Pesquisa do Estado de São Paulo (FAPESP).

(1) Mestre em Oftalomologia pela Universidade Federal de São Paulo - EPM.

(2) Livre Docente pela Universidade Federal de São Paulo - EPM.

(3) Doutores em Medicina pela Universidade Federal de São Paulo - EPM.

Endereço para correspondência: Paulo Henrique Morales - Rua Ribeiro de Barros, 266 - São Paulo (SP) CEP 05027-020. E-mail: phmorales@oftalmologista.com.br

Nota Editorial: Pela análise deste trabalho e por sua anuência sobre a divulgação desta nota, agradecemos a Dr. Walter Y. Takahashi.

\footnotetext{
\begin{tabular}{l} 
INTRODUÇÃo \\
\hline A cirurgia refrativa apresentou um grande avanço neste final de século, \\
e com isso ocorreu a popularização de suas diversas modalidades. Quem \\
mais se beneficiou com este tipo de cirurgia foram os míopes ${ }^{1}$. \\
A miopia é uma das cinco maiores causas de cegueira legal em todo \\
mundo ${ }^{1}$. O déficit visual ocorre em idade precoce, comparado com a faixa \\
etária das outras quatro principais causas: catarata, retinopatia diabética, \\
glaucoma e degeneração macular relacionada à idade, que ocorrem décadas \\
depois. Do ponto de vista social, a miopia causa um enorme impacto, pois \\
pode alterar o desempenho de indivíduos na etapa produtiva de suas vidas ${ }^{1}$. \\
Dentre as causas de cegueira associadas a olhos míopes predominam as
\end{tabular}
} 
alterações do segmento posterior do bulbo ocular, como: descolamento regmatogênico da retina, alterações degenerativas maculares, estafilomas posteriores com ectasia macular, neovascularização subretiniana macular, buraco de mácula e glaucoma ${ }^{2}$.

Estes olhos apresentam maior risco e em idade mais precoce de desenvolver descolamento regmatogênico de retina que a população geral, por causa direta, por uma deficiência da matriz que une os fotorreceptores ao epitélio pigmentado retiniano e/ou no mecanismo de bomba do epitélio pigmentado retiniano; ou por forma indireta, pelo estiramento da retina deixando-a susceptível a lesões degenerativas e roturas, causadas por uma menor resistência à tração vítreo-retiniana ${ }^{3-6}$.

O descolamento regmatogênico está associado às degenerações periféricas de retina, as quais são identificadas por meio da observação clínica, podendo ser classificadas pela morfologia, prognóstico e predisposição ou não ao descolamento regmatogênico de retina ${ }^{7}$.

No presente estudo procurou-se determinar a freqüência de lesões degenerativas da periferia da retina, predisponentes ou não ao desenvolvimento de descolamento de retina, em pacientes candidatos à cirurgia de miopia e relacioná-las com o tipo de miopia.

\section{MÉTODOS}

Dos participantes do estudo foram obtidos os dados de idade, sexo, sintomas relacionados à fotopsia e antecedentes pessoais e familiares de descolamento regmatogênico de retina. O exame oftalmológico constou de: biomicroscopia, refração estática e oftalmoscopia indireta com depressão escleral circunferencial. A biomicroscopia de fundo de olho com lente de três espelhos foi efetuada nos casos onde houve dificuldade de caracterização das lesões periféricas da retina pela oftalmoscopia indireta. A midríase foi realizada com colírio de ciclopentolato a $1 \%$ e fenilefrina a $10 \%$.

Neste estudo foi usada a classificação de $\mathrm{Otsuka}^{8}$, que relaciona o grau de ametropia com as alterações retinianas anatômicas e estruturais, que segundo o exame de refração estática divide a miopia em 3 categorias dependendo do equivalente esférico: Miopia baixa $(-1,00$ e $-3,00$ dioptrias esféricas), Miopia moderada (-3,25 e -6,00 dioptrias esféricas.) e Miopia alta (superior a $-6,00$ dioptrias esféricas).

Todos os exames oftalmológicos completos foram realizados pelo mesmo examinador; os pacientes com lesões periféricas de retina foram reexaminados por dois outros examinadores que confirmaram ou não a existência das lesões.

Para verificar possíveis associações entre os graus de miopia e os vários tipos de lesões, foi utilizado o teste da decomposição aditiva do Qui-quadrado $\left(\mathrm{x}^{2}\right)$ nas tabelas de associação. Foram obedecidas as restrições de Cochran, sendo que as tabelas não analisáveis apresentaram ressalvas.
Fixou-se como nível de rejeição de hipótese de nulidade o valor $0,05(5 \%)$.

\section{RESULTADOS}

Foram examinados 840 olhos míopes de 432 pacientes, sendo 233 do sexo feminino e 199 pacientes do sexo masculino. A média de idade da população estudada foi de 31 anos variando de 17 a 66 anos.

De acordo com a classificação dos pacientes segundo o tipo de miopia, foram identificados $314(73 \%)$ pacientes apresentando o mesmo tipo de miopia em ambos os olhos, 94 (22\%) com classificação diferente de miopia nos dois olhos e 24 (5\%) apresentaram algum tipo de miopia em apenas um dos olhos.

A miopia baixa foi detectada em 167 pacientes (Figura 1). Esse tipo de miopia ocorreu em ambos os olhos em 96 pacientes e em apenas um dos olhos em 71 pacientes, perfazendo um total de 263 (31\%) olhos.

A miopia moderada foi identificada em 195 pacientes (Figura 1), sendo em ambos os olhos em 105 pacientes e em apenas um dos olhos em 90 pacientes, somando 300 (36\%) olhos.

A miopia alta, identificada em 164 pacientes (Figura 1), era bilateral em 113 e unilateral em 51 pacientes, totalizando 277 (33\%) olhos.

Em 225 (27\%) olhos foram encontradas lesões periféricas de retina, sendo que em 63 (8\%) olhos havia lesões predisponentes ao descolamento regmatogênico de retina e em 35 (4\%) olhos foram observadas lesões predisponentes e não predisponentes ao descolamento regmatogênico de retina, totalizando $98(12 \%)$ olhos com lesões predisponentes ao descolamento regmatogênico de retina. Em 127 (15\%) registraram-se apenas lesões não predisponentes ao descolamento regmatogênico de retina (Figura 2).

A degeneração em treliça identificada em 50 (6\%) olhos (Figura 3) apresentou diferença em prevalência, quando analisamos o grupo de portadores de miopias baixa e alta, constatandose uma proporção de 1:2,2 entre os grupos. Para ambos os sexos a degeneração em treliça foi mais comum nos olhos com alta miopia e a bilateralidade foi mais freqüente na miopia moderada.

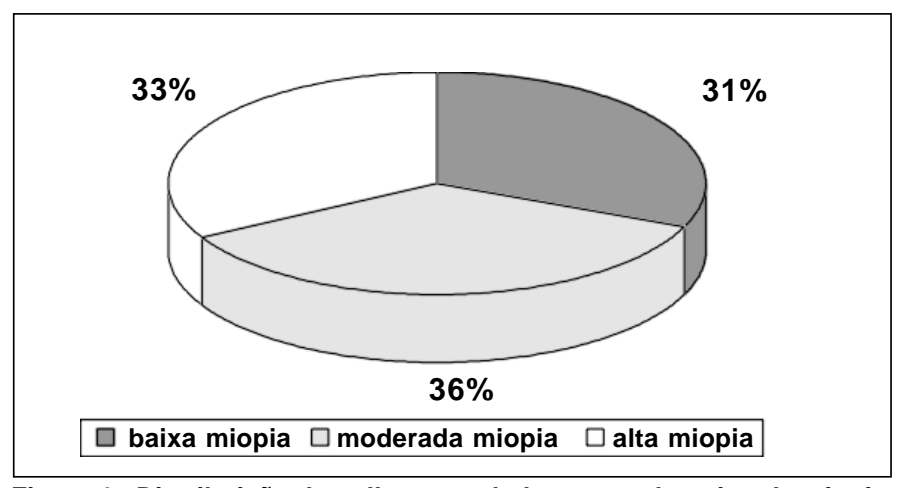

Figura 1 - Distribuição dos olhos estudados segundo o tipo de miopia 


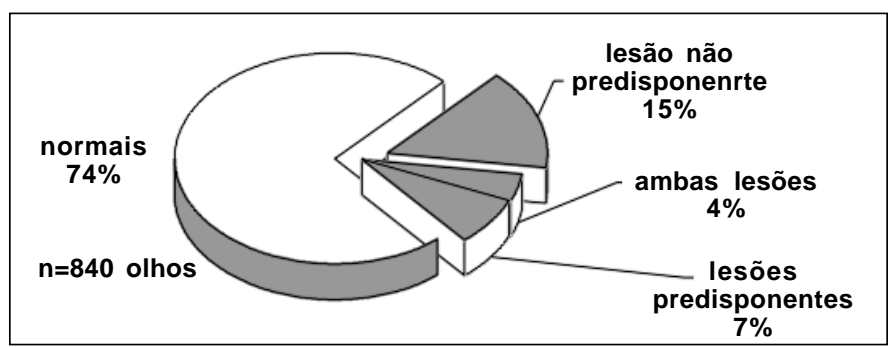

Figura 2 - Indentificação das lesões encontradas ao mapeamento da retina expressa em porcentagem

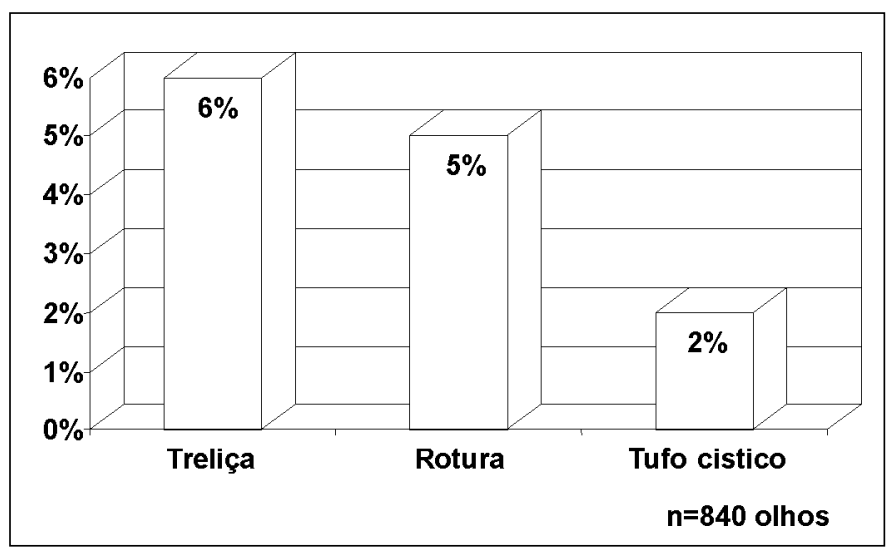

Figura 3 - Distribuição das lesões prediponentes ao descolamento de retina encontradas nos olhos examinados

A presença de roturas da retina (Figura 3) ocorreu em menor número nos olhos com miopia moderada, sendo a freqüência 2,4 vezes maior nas miopias baixas e três vezes maior nas miopias altas; esta tendência ocorreu independente do sexo. Houve uma tendência das roturas serem unilaterais e essa tendência foi menor nas miopias altas.

Os tufos císticos de retina (Figura 3) encontrado com maior freqüência nas miopias altas do que nas moderadas ou baixas, 3 e 3,5 vezes respectivamente, e em $2,1 \%$ do total de olhos. Essa tendência ocorreu em ambos os sexos. Como a degeneração em treliça, a miopia baixa tendeu a ser unilateral.

Do total de 162 olhos que apresentavam lesão não predisponente ao descolamento regmatogênico de retina, 147 $(17,5 \%)$ possuíam branco com ou sem pressão, $16(1,9 \%)$ tinham degeneração pavimentosa e a retinosquise não foi encontrada em nenhum dos pacientes. A freqüência dessas degenerações demonstrou uma relação direta com o tipo de miopia.

A degeneração branco com ou sem pressão foi 2,3 e 3,5 vezes mais comum nas miopias moderada e alta, respectivamente, do que na baixa. Essa prevalência progressiva com o grau de miopia foi estatisticamente significante em ambos os sexos. Como a maioria das outras degenerações, o branco com ou sem pressão teve a tendência a ser bilateral nos tipos de miopia moderada e alta.

A degeneração em pedras de calçamento foi observada 2,2 e 4,3 vezes mais nas miopias moderada e alta, respectivamente, do que na baixa. Essa mesma tendência ocorreu em ambos os sexos.

\section{DISCUSSÃO}

Cinco a dez por cento dos casos de descolamento regmatogênico de retina, após tratamento cirúrgico, apresentam complicações pós-operatórias variáveis ${ }^{9}$. Do total de casos com êxito anatômico pós-operatório cerca de $40 \%$ tem acuidade visual final melhor que 20/50 ${ }^{9-10}$. Assim, devido às graves conseqüências do descolamento regmatogênico de retina, dos pontos de vista tanto anatômico quanto funcional, os esforços devem se concentrar na profilaxia e no tratamento precoce antes do acometimento macular, fator de prognóstico funcional.

A prevenção do descolamento regmatogênico de retina poderia ser feita de três formas: 1) evitar a liquefação vítrea e o descolamento posterior do vítreo; 2) aliviar a tração vítreoretiniana e 3) localizar e tratar de acordo com critério de risco as lesões retinianas predisponentes com potencial para causar descontinuidades teciduais da retina, precursoras do descolamento regmatogênico de retina ${ }^{10}$.

No entanto não há como evitar a liquefação vítrea, visto que ela é parte das alterações que ocorrem com o envelhecimento ${ }^{6,11}$. A tração vítreo-retiniana pelas complicações e impossibilidade técnica de (no caso da vitrectomia) retirar o vítreo anterior-periférico nos olhos fácicos (sendo este o principal fator na formação de novas roturas), são aliviadas apenas em casos específicos, onde existam tração e alto risco de descolamento regmatogênico de retina ${ }^{12}$.

O descolamento regmatogênico de retina afeta uma pequena percentagem da população geral, sendo possível estabelecer com base nos dados colhidos por este estudo as faixas etárias com risco para descolamento, dependendo do erro refrativo e da presença ou ausência de degenerações periféricas retinianas ${ }^{13}$.

Aproximadamente $1 / 3$ da população desse estudo apresentava miopia alta, com uma média de idade de 31 anos, próxima à faixa etária em que o descolamento regmatogênico de retina ocorre com maior freqüência. Isso quer dizer que, dos pacientes que se apresentaram no Setor de Cirurgia Refrativa, $1 / 3$ pertence ao grupo de risco para desenvolvimento de descolamento regmatogênico de retina somente pela história natural dessa doença, mesmo que nenhum procedimento seja realizado ${ }^{13}$.

A retinosquise não foi observada provavelmente devido ao fato dessas lesões ocorrerem, em geral, em pacientes idosos e hipermétropes ${ }^{14-15}$ - característica ausente no grupo em estudo.

O branco com e sem pressão que representa um conjunto de alterações gerais inespecíficas que apresentam um mesmo aspecto clínico na retina, foi a lesão mais encontrada. Nossas 
observações de que nas miopias moderada e alta, o branco com e sem pressão é 2,3 e 3,5 vezes mais comum, respectivamente, do que na miopia baixa, são compatíveis com a literatura ${ }^{2}$, o qual afirma que a freqüência dessa alteração está diretamente relacionada com o grau de miopia. A presença do branco com e sem pressão não influi no risco para descolamento regmatogênico de retina ${ }^{2,16}$, a não ser no olho contralateral de pacientes com rotura gigante ${ }^{12}$.

A degeneração pavimentosa não está associada ao descolamento regmatogênico de retina ${ }^{17}$. Apesar desse estudo estar em concordância com a literatura de que a freqüência dessa alteração está diretamente relacionada ao grau de miopia ${ }^{2}$, pois essa alteração é 2,2 e 4,3 vezes mais comum nas miopias moderada e alta, respectivamente, do que na baixa, a prevalência deste estudo esteve abaixo da encontrada em grandes séries de necropsia ${ }^{18}$. Esse fato pode ser explicado pela relação dessa degeneração com a idade ${ }^{19}$.

A degeneração em treliça (Figura 4) foi encontrada em uma proporção de 1:2,2 entre os grupos portadores de miopias baixa e alta, apesar de os relatos de Shiomi ${ }^{21}$ e Celorio \& Pruett (1991) sugerirem uma relação indireta entre o grau de miopia e a presença de degeneração em treliça, isto é, quanto maior o equivalente esférico, menor a prevalência da lesão. Nessa amostra houve aumento da prevalência dessa lesão diretamente relacionado ao grau de miopia, o que está de acordo com a literatura ${ }^{19,22-24}$.

Pelos relatos de Straatsma et al. ${ }^{24}$ a incidência dessa lesão já é máxima na segunda década de vida e novas lesões podem ocorrer em apenas 0,25\% dos casos após os 19 anos.

Pelos estudos de Byer ${ }^{22}$, sabe-se que a miopia é um fator de risco para o descolamento regmatogênico de retina; quando se comparam pacientes míopes e não míopes, verificase que o descolamento regmatogênico de retina é de 3 a 5 vezes mais freqüente nos míopes e 15 vezes mais freqüente nas miopias altas. A presença de degeneração em treliça aumenta a possibilidade de descolamento regmatogênico de retina em dez vezes. Um alto míope com essa degeneração apresenta 150 a 200 vezes mais chances de desenvolver descolamento regmatogênico de retina do que um paciente não míope ${ }^{4}$. Portanto, estima-se que $3,24 \%$ dos pacientes desse estudo com miopia alta e degeneração em treliça, bem

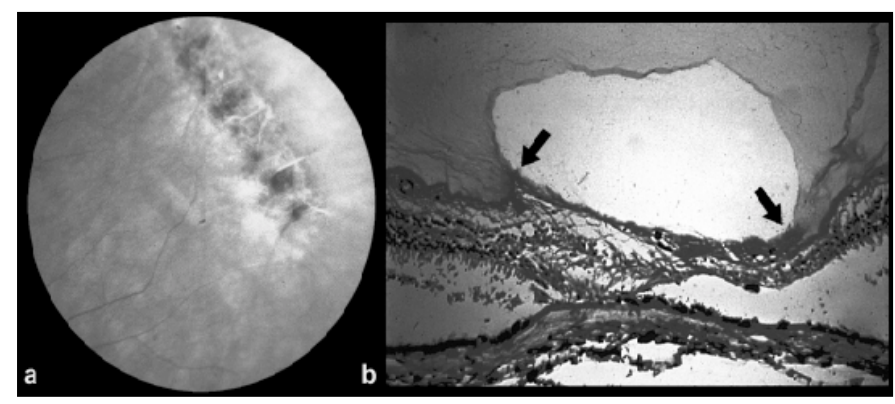

Figura 4 - Degeneração em treliça considerada lesão predisponenete ao descolamento de retina como $0,4 \%$ daqueles com miopia baixa ou moderada e degeneração em treliça desenvolverão descolamento regmatogênico de retina.

As roturas (Figura 5) estão associadas ao descolamento regmatogênico de retina nas baixas miopias ${ }^{4}$. A prevalência dessas alterações nos pacientes desta pesquisa $(4,3 \%)$ foi próxima à mencionada por $\operatorname{Byer}^{25}(8,7 \%)$ e registrada por Foos ${ }^{11}$ $(1,9 \%)$ em grandes amostras de necropsia.

A incidência de roturas varia com a idade, sendo maior na $4^{\mathrm{a}}, 5^{\mathrm{a}}$ e $6^{\mathrm{a}}$ décadas de vida ${ }^{16,26-27}$, o que permite esperar um aumento do número de roturas nos pacientes deste estudo com o passar dos anos. Portanto, o acompanhamento desses pacientes no pós-operatório é importante, bem como observar novas roturas e sua eventual relação com procedimentos a que foram submetidos.

Neste estudo as roturas de retina foram observadas em menor número nos olhos com miopia moderada, sendo a prevalência 2,4 vezes maior nas miopias baixas e três vezes maior nas miopias altas. $\mathrm{O}$ mesmo foi observado no trabalho de Pierro et al. ${ }^{28}$, em que relacionaram apenas pacientes míopes, encontrando maior prevalência de roturas nas miopias baixas e altas ${ }^{29}$.

Byer em estudo de 223 olhos com roturas retinianas e buracos com ou sem opérculo em pacientes fácicos, assintomáticos e sem outra degeneração retiniana associada, observou que nenhum deles desenvolveu descolamento regmatogênico de retina por aproximadamente sete anos e meio ${ }^{16}$. Neste trabalho, o grupo com roturas de retina apresentou as mesmas características dos critérios de elegibilidade usados no trabalho de Byer (1982) o que leva a acreditar que estes pacientes apresentem chance pequena de desenvolver descolamento regmatogênico de retina.

Davis estudando 176 olhos não tratados, com roturas retinianas ou com descolamento subclínico (definido como

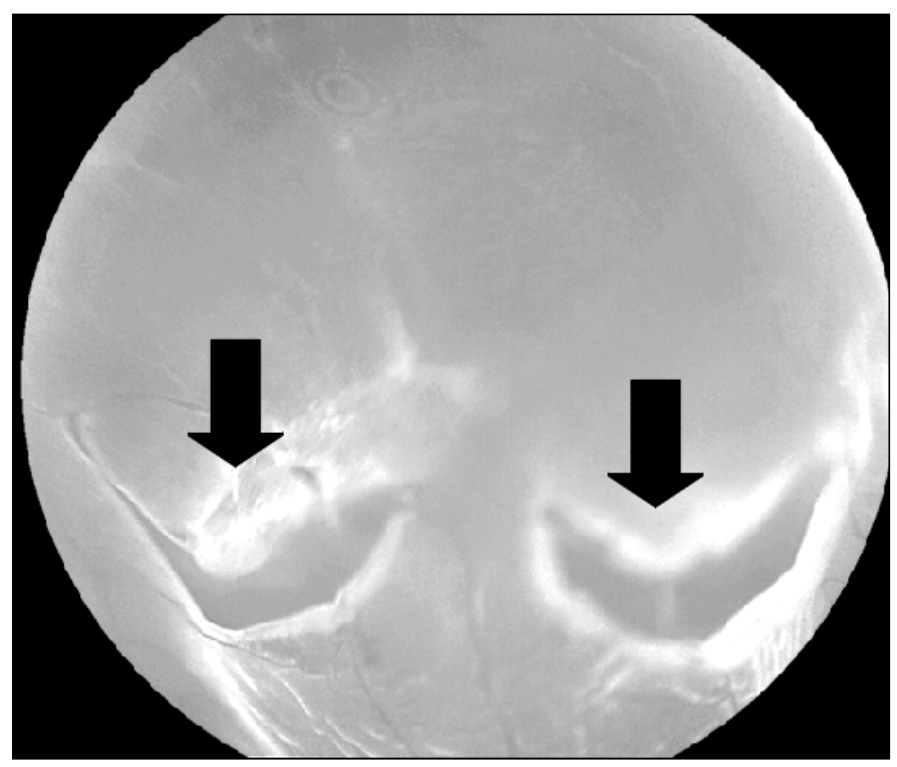

Figura 5 - Descontinuidades de retina tipo rotura 


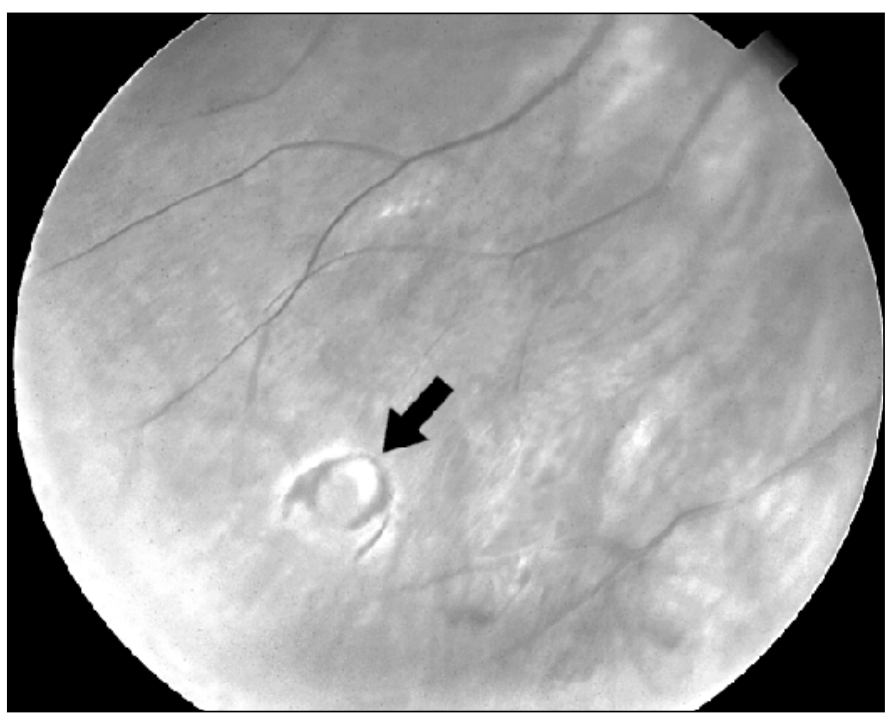

Figura 6 - Descontinuidade de retina associada a tufo cístico de retina

descolamento regmatogênico de retina de 1 a 2 diâmetros de papila em torno da rotura), referiu que $18 \%$ tiveram progressão para descolamento regmatogênico de retina, identificando três grupos em que ocorreu esta progressão: 1) olhos sintomáticos com roturas acima do meridiano horizontal; 2) olhos com roturas associadas ao descolamento subclínico; 3 ) olhos afácicos com roturas, incluindo principalmente os que apresentavam descontinuidade retiniana antes da facectomia. A percentagem de descolamento nesses grupos foi 35, $30 \mathrm{e}$ $50 \%$, respectivamente. Estes fatores podem estar presentes na cirurgia dos altos míopes ${ }^{30}$.

Outra lesão considerada predisponente ao descolamento regmatogênico de retina é o tufo cístico de retina que é um remanescente embriológico, caracterizando-se por uma projeção de tecido retiniano, branco-acinzentado, de aproximadamente $1 / 4$ de diâmetro de papila, anterior ao equador do olho, próximo a ora serrata, com traves vítreas aderidas em seu ápice, tornando-as potencialmente predisponentes para o desenvolvimento de roturas por tração vítrea e descolamento regmatogênico de retina.

Devemos recordar que apesar do tufo cístico de retina estar em grande parte relacionado aos buracos com opérculos livres ${ }^{11}$, são os cistos relacionados às roturas em ferradura que levam ao descolamento regmatogênico de retina ${ }^{16}$.

É no grupo dos míopes altos onde essa degeneração é mais preocupante, pois é neste grupo em que cirurgias de miopia podem induzir o descolamento posterior de vítreo.

Barraquer et al., em 165 olhos de 107 pacientes submetidos à extração de cristalino e implante de lente intra-ocular para correção de sua miopia, encontravam 7,27\% apresentaram descolamento regmatogênico de retina em média 30 meses após a cirurgia sendo que destes apenas $25 \%$ apresentaram descolamento regmatogênico de retina nos primeiros 6 meses. No mesmo levantamento foi estimado que pacientes com menos de 30 anos tinham 2,5 vezes mais chances de descolamento regmatogênico de retina que os pacientes acima dessa idade e, naqueles em que foram submetidos à capsulotomia posterior apresentaram 2 vezes mais descolamento regmatogênico de retina que os com cápsula posterior intacta ${ }^{4}$.

Assim, é possível que o descolamento regmatogênico de retina após cirurgias em pacientes com miopia alta, onde a câmara anterior é violada, é causado por alterações fisiopatológicas secundárias, particularmente do corpo vítreo, deste olho e não por uma ação direta da cirurgia. Freqüência semelhante foi encontrada em trabalhos realizados por Coonan et al. ${ }^{31}$ e Lindstrom et al. ${ }^{32}$, onde a incidência de descolamento regmatogênico de retina após facectomia em pacientes com miopia alta foi respectivamente de 3,5 e 9,6\%.

É o grupo de miopia alta que traz maior preocupação ao oftalmologista pois além das degenerações, as cirurgias de miopia podem induzir ou propiciar o aparecimento do descolamento posterior de vítreo ${ }^{29}$.

Ao contrário das cirurgias que invadem a câmara anterior para correção de miopia, acredita-se que o descolamento regmatogênico de retina nas cirurgias não invasoras, como ceratotomia radial e fotoablação, é decorrente da história natural dos olhos míopes ou dos efeitos do uso de mióticos no pré e/ou pós-operatórios desses pacientes ${ }^{29,33}$.

Deve-se continuar essa linha de pesquisa no sentido de identificar os grupos com necessidade de tratamento profilático nas degenerações periféricas de retina e evitar o descolamento regmatogênico de retina. Pela complexidade dos fatores que propiciam o descolamento regmatogênico de retina, as cirurgias refrativas devem ser estudadas separadamente com análise pré e pós-operatória para identificar suas reais contribuições e os grupos onde estes fatores ocorrem.

Este estudo nos possibilita concluir que os pacientes que procuram o serviço de cirurgia refrativa são jovens (média de 31 anos); um terço dos pacientes são míopes altos (Figura 1); próximo da metade dos pacientes apresentam lesões periféricas de retina (Figura 2).

O profissional que examina esses pacientes deve estar familiarizado com a diferenciação entre lesões predisponentes e não predisponentes, pois em $44 \%$ das lesões esse diagnóstico vai ser importante (Figura 2 ).

A degeneração em treliça foi a lesão predisponente ao descolamento regmatogênico de retina mais freqüente e com exceção do buraco de retina, todas as outras degenerações aumentaram a sua freqüência com o aumento do grau de miopia.

O cirurgião de refrativa trabalha com um grupo de pacientes com predisposição ao descolamento regmatogênico de retina. Nos pacientes míopes que serão submetidos à cirurgia refrativa, com procedimento intra-ocular ou que necessitem usar colírios mióticos, a cuidadosa inspeção da periferia retiniana deve ser obrigatória, pela prevalência de degeneração em treliça, buracos e tufo cístico de retina. 


\section{ABSTRACT}

Purpose: To verify, in myopic individuals who are candidates for refractive surgery, the prevalence of different types of peripheral degenerative lesions of the retina, according to the type of myopia. Methods: Prospectively, during a one-year interval, we examined the eyes of patients in the Refractive Surgery Sector of the Department of Ophthalmology of the Federal University of São Paulo - Paulista School of Medicine, who presented at the initial visit a refraction with spherical equivalent above or equal to -1.00 spherical diopter, and did not have a personal background of ocular disease or surgery in that period. We investigated the existence of lesions and/or peripheral degeneration, which predispose to rhegmatogenous detachment of the retina. Results: The group was mostly composed of young adults (average age 31). We observed eyes with low myopia (263 eyes, 31\%), moderate myopia (300 eyes, 36\%) and high myopia (277 eyes, 33\%). In $35.4 \%$ of the patients $(27 \%$ of the eyes) we found peripheral degeneration, and the white with or without pressure was the most frequent finding $(23.4 \%$ of the patients or $17.5 \%$ of the eyes). Among the lesions that predispose to rhegmatogenous detachment of the retina, the most frequently found was the lattice degeneration (8.6\% of the patients or $6 \%$ of the eyes). Conclusions: The peripheral alterations which predispose or not to rhegmatogenous detachment of the retina presen- ted an increase in prevalence according to the increase in the myopia grade, with the exception of tears. All patients with high myopia and candidates for refractive surgery should have the retinal periphery of both eyes examined.

Keywords: Retinal detachment; Radial keratotomy; Photorefractive keratectomy excimer laser/adverse effects; Myopia/surgery.

\section{REFERÊNCIAS}

1. Waring GO. Myopia. In: Myopia: surgery. St. Louis; Mosby, 1992. p. 4-15.

2. Curtin BJ. The myopias: basic science and clinical management. New York: Harper \& Row; 1985.

3. Austin KL, Palmer JR, Seddon JM, Glynn RJ, Rosenberg L, Gragoudas ES, et al. Case control study of idiopathic retinal detachment. Int J Epidemiol 1990;19:1045-50.

4. Barraquer C, Cavelier C, Mejia L. Incidence of retinal detachment following clear-lens extraction in myopic patients. Arch Ophthalmol 1994;112:338-9.

5. Ogawa A, Tanaka M. The relationship between refractive errors and retina detachment: analysis of 1166 retinal detachment cases. Jpn J Ophthalmol, 1988;32:310-5.

6. Sebag J. Aging of the vitreous. Eye, 1987;1:254-62.

7. Kansky JJ. The classification and terminology of peripheral retinal degenerations. Mod Probl. Ophthalmol 1975;15:103-11.

8. Otsuka J. Research on the etiology and treatment of myopia. Acta Soc Ophthalmol Jpn 1967;71:13.

9. Hilton GF, McLean EB, Chuang EL. Pathogenesis and natural history. In: Hilton GF, McLean EB, Brinton DA. Retinal detachment: principles and practice. $2^{\text {nd }}$ ed. San Francisco: American Academy of Ophthalmology; 1995. p. 7-37.

10. Michels RG, Wilkinson CP, Rice TA. Retinal detachment. St. Louis: Mosby; 1997.

11. Foos RY. Postoral peripheral retinal tears. Ann. Ophthalmol 1974;6:679-87.

12. Freeman HM. Fellow eyes of giant retinal breaks. Trans Am Ophthalmol Soc 1978;76:343-82.

13. Burton TC. The influence of refractive error and lattice degeneration on the incidence of retinal detachment. Trans Am Ophthalmol Soc 1989;87:143-57.

14. Byer NE. Clinical study of retinoschisis. Arch Ophthalmol 1968;79:36-44.

15. Tolentino FI, Schepens CL, Freeman HM. Vitreous detachment. In: Tolentino FI. Vitreoretinal disorders: diagnosis and management. Philadelphia: Saunders; 1976. p. 130-54.

16. Byer NE. The peripheral retina profile: a stereoscopic atlas. Torrance: Criterion Press; 1982.

17. Straatsma BR, Foos RY, Freman SS. Degenerative diseases of the peripheral retina. In: Duane TD: Clinical ophthalmology. Philadelphia: Haper \& Row; 1986. v.3, p. 1.

18. O'Malley P, Allen PA, Straatsma BR et al. Paving-stone degeneration of the retina. Arch Ophthalmol 1965;73:169-82.

19. Karlin DB, Curton BJ. Peripheral chorioretinal lesions and axial length of myopic eye. Am J Ophthalmol 1976;81:625-35.

20. Shiomi Y. Study of Lattice degeneration of the retina. Part 2. Clinical feature of Lattice degeneration of the retina. Acta Soc Ophthalmol Jpn 1981;85:269-75.

21. Celorio JM, Pruett RC. Prevalence of lattice degeneration and its relation to axial length in severe myopia. Am J Ophthalmol 1991;111:20-3.

22. Byer NE. Lattice degeneration of the retina. Surv Ophthalmol 1979;23:213-48.

23. Morse PH, Scheie HG, Aminlari A. Light flashes as a clue to retinal disease. Arch Ophthalmol 1974;91:179-80.

24. Straatsma BR, Zeegen PD, Foos RY, Feman SS, Shabo AL. XXX Edward Jackson Memorial Lecture. Lattice degeneration of the retina: Trans Am Acad. Ophthalmol Otolaryngol 1974;78:OP87-113.

25. Byer NE. Clinical study of retinal breaks. Trans Am J Ophthalmol Otolaryngol 1967;71:461-73.

26. Foos RY, Wheelers NC. Vitreoretinal juncture. Synchysis senilis and posterior vitreous detachment. Ophthalmology 1982;89:1502-12.

27. Hyams SW, Neumann E. Peripheral retina in myopia with particular reference to retinal breaks. Br J Ophthalmol 1969;53:300-6.

28. Pierro L, Camesasca FI, Mischi M, Brancato R. Peripheral retinal changes and axial myopia. Retina 1992;12:12-7.

29. Rodriguez A, Camacho H. Retinal detachment after refractive surgery for myopia. Retina 1992;12(3Suppl):46-50.

30. Davis MD. The natural history of retinal breaks without detachment. Trans Am Ophthalmol Soc 1973;71:342-72.

31. Coonan P, Fung WE, Webster RG Jr., Allen AW, Abbott RL. The incidence of retinal detachment following extracapsular cataract extraction: a ten-year study. Ophthalmology 1985;92:1096-101.

32. Lindstrom RL, Lindquist TD, Huldin J, Rubenstein JB. Retinal detachment in axial myopia following extracapsular cataract surgery. Trans New Orleans Acad Ophthalmol 1988;36:263-8.

33. Hofman RF, Starling JC, Hovland KR. Case report: retinal detachment after radial keratotomy surgery. J Refract Surg 1985;1:226.

\title{
Novidades na Internet!!!
}

\author{
Agora no site CBO você tem disponivel todas as informações na íntegra dos Arquivos Brasileiros de Oftalmologia \\ http://www.cbo.com.br/abo
}

